\section{Convergence of Iterative Water-Filling With Quantized Feedback: A Sufficient Condition}

Ramy H. Gohary and Halim Yanikomeroglu

\begin{abstract}
In this paper, the iterative water-filling (IWF) algorithm is considered when the noise-plus-interference levels fed back from the receivers are quantized. A sufficient condition for this algorithm to have a unique Nash equilibrium is derived. This condition is shown to approach the corresponding condition for standard IWF when the quantization resolution or the transmission power are sufficiently high.
\end{abstract}

Index Terms-Convergence, nonnegative matrix, noncooperative games, Nash equilibrium, power allocation.

\section{INTRODUCTION}

I N OPEN spectrum communication systems several user pairs share a common bandwidth of multiple orthogonal subchannels. The users organize their transmissions autonomously without central coordination. Given a total power budget, each user allocates its power across the available subchannels in such a way that maximizes its own utility, rather than the network utility. The transmissions of each user must meet a spectral mask constraint that ensures that the power allocated to each subchannel does not exceed a prescribed threshold. When the utility is given by the rate at which data can be reliably decoded, users may employ the so-called iterative water-filling (IWF) algorithm [1]. In standard IWF, the receivers measure their observed noise-plus-interference (NI) levels on all subchannels and feed back this information to their respective transmitters. Based on the received NI levels the transmitters update the power allocation across the subchannels in such a way that maximizes their achievable data rate.

When a user updates its power allocation, the NI levels observed by other users in the network change, causing them to update their power allocations accordingly to maximize their achievable data rates. This situation can be modelled as a noncooperative game in which users are represented by competitive players and feasible power allocations are represented by admissible strategies [2], [3].

In a practical communication system, it is desirable for the power allocation game to have a unique Nash equilibrium; that is, a unique set of power allocations at which no user can attain a higher rate by making unilateral changes to its power allocation strategy. When such an equilibrium is reached and the channel is static, feeding back the NI levels to the transmitters may not be necessary and the transmitters do not need to update their codebooks to adapt to fluctuations in the rate that can be supported by varying power allocations. Furthermore, the existence of a unique Nash equilibrium implies that the equilibrium communication rates do not depend on the initial power allocations and can be determined a priori. This enables the codebooks to be designed offline. In contrast, when the game possesses multiple Nash equilibria, the equilibrium rates and power allocations will depend on the initial

Manuscript received December 26, 2010; revised April 19, 2011 and October 12, 2011; accepted February 13, 2012. Date of publication March 08, 2012; date of current version April 13, 2012. This associate editor coordinating the review of this manuscript and approving it for publication was Prof. Ye (Geoffrey) Li. This work was supported in part by Research In Motion, and also by the Collaborative Research and Development (CRD) Grant of the Natural Sciences and Engineering Research Council (NSERC) of Canada.

The authors are with the Department of Systems and Computer Engineering, Carleton University, Ottawa, ON K1S 5B6 Canada (e-mail: gohary@sce.carleton.ca; halim@sce.carleton.ca).

Digital Object Identifier 10.1109/TSP.2012.2188715 power allocations, and determining these rates and the corresponding codebooks becomes less straightforward. It is to be noted that, due to the selfish nature of the IWF algorithm, the power allocations it generates, even at equilibrium, are not necessarily Pareto optimal [4]. In fact, power allocations that yield uniformly higher rates might be achievable if the users were to select their power allocations cooperatively.

Sufficient conditions under which the power allocation game converges to a unique Nash equilibrium have been derived in [1] and [2] for synchronous single antenna systems when exact NI levels are available at the transmitters. These results were extended in [3] to precoded multiple-input multiple-output systems with finite-size constellations and in [5] to asynchronous systems in which the users update their power allocations using outdated NI level information. In addition to those selfish power allocation strategies, a more cooperative approach was proposed in [6]. In this approach, the users exchange their power prices in order to maximize the overall network utility rather than their own utilities. Being cooperative, the rates obtained by this price-based IWF can be regarded as an upper bound on the rates obtained by competitive schemes including the standard IWF and the quantized IWF (QIWF) considered herein.

In IWF-based algorithms, the assumption that exact NI levels are available at the transmitters is not realistic, because, in practice, only a small number of bits is used for feeding back these levels. Unless properly accounted for, the uncertainty in the NI levels can lead the transmitters to transmit data at rates that are not decodable by the receivers. To overcome this difficulty, robust algorithms based on IWF have been proposed in [7] and [8]. Using the algorithm in [7], it was shown that uncertainty can be beneficial if it is properly accounted for by scaling the NI levels. In particular, by adjusting the quantization resolution at the receivers and the scaling factor at the transmitters, it was shown in [7] that the performance of the robust IWF algorithm approaches that of the cooperative price-based IWF one [6].

Despite being inherent in the practical operation of the IWF algorithm, NI quantization has not been accounted for in currently available convergence analyses, e.g., [1]-[3], and [8]. It is, therefore, important to question the relevance of these analyses in that practical case. In particular, the question that we ask here is: How does the quantization of the NI levels impact convergence? To answer this question in the case of synchronous single antenna systems, we provide a novel technique that relies on representing the quantization noise by equivalent iteration-dependent channel coefficients. By proper bounding of these coefficients, we derive a sufficient condition that ensures that the quantized power allocation game has a unique Nash equilibrium to which the iterates are guaranteed to converge. Drawing insight from this condition, it is shown that if the IWF algorithm with exact NI levels satisfies the convergence conditions in [2], the QIWF algorithm also converges provided that the quantization resolution or the users' powers are sufficiently high. Furthermore, it is shown that, when the transmitters scale the NI levels to account for quantization uncertainty as proposed in [7], the convergence condition becomes more stringent.

In Section II, the system model and the problem statement are presented. In Section III, the convergence condition is derived. Section IV provides numerical examples, and Section $\mathrm{V}$ concludes the paper.

\section{SySTEM MODEL AND PROBLEM STATEMENT}

Consider a wireless system with $K$ user pairs and $N$ quasi static orthogonal subchannels. Each user pair has one transmit and one receive antenna. The received signal of user $k$ on the $n$th subchannel is given by: $y_{k}^{n}=h_{k k}^{n} x_{k}^{n}+\sum_{j=1, j \neq k}^{K} h_{j k}^{n} x_{j}^{n}+v_{k}^{n}$, where $h_{j k}^{n}$ is the complex channel gain between the transmitter of user $j$ and the receiver of user $k$ on the $n$th subchannel, $j, k \in \mathcal{K}=\{1, \ldots, K\}$, 
$n \in \mathcal{N}=\{1, \ldots, N\}, x_{k}^{n}$ is the transmitted signal of user $k$ on the $n$th subchannel, and $v_{k}^{n} \sim \mathcal{C N}\left(0, N_{0}\right)$ is the additive white Gaussian noise observed by the receiver of user $k$ on the $n$th subchannel. Each user $k \in \mathcal{K}$ has a total power budget $P_{k}$, which can be distributed across the $N$ subchannels, provided that the power allocated to any subchannel $n \in \mathcal{N}$ does not exceed a prescribed spectral mask $S_{\max , k}^{n}$.

In the next section we will revisit the standard IWF algorithm, and in Section II-B we will extend this algorithm to the case in which the NI levels are quantized.

\section{A. Iterative Water-Filling (IWF)}

In the standard IWF algorithm [1], each user can decode its intended signals, but not the signals intended for other users. Hence, each user observes the signals intended for other users as additive interference. The receivers measure their observed noise-plus-interference (NI) levels on each subchannel and feed back these levels to their respective transmitters. Assuming that all users use Gaussian signalling, the transmitter of each user determines a power distribution across the subchannels that enables it to achieve the maximum data rate. The users update their powers iteratively until equilibrium is reached.

Let $\alpha_{j k}^{n}$ be the normalized square channel gain of the $n$th subchannel between user $j$ and user $k$; i.e., $\alpha_{j k}^{n}=\left|h_{j k}^{n}\right|^{2} /\left|h_{k k}^{n}\right|^{2}$. Let $s_{k}^{n,(\nu)}=$ $\mathrm{E}\left\{\left|x_{k}^{n}\right|^{2}\right\}$ denote the power allocated by user $k \in \mathcal{K}$ to subchannel $n \in$ $\mathcal{N}$ at the $\nu$ th iteration, where $\mathrm{E}\{\cdot\}$ denotes the expectation operator.

Assuming that the users update their power sequentially, the normalized NI level observed by user $k$ on the $n$th subchannel at the $\nu$ th iteration is given by

$$
I_{k}^{n,(\nu)}=\sigma_{k}^{n}+\sum_{j=1}^{k-1} \alpha_{j k}^{n} s_{j}^{n,(\nu)}+\sum_{j=k+1}^{K} \alpha_{j k}^{n} s_{j}^{n,(\nu-1)}
$$

where $\sigma_{k}^{n}=N_{0} /\left|h_{k k}^{n}\right|^{2}$ is the equivalent noise variance [2], [7], and $N_{0}=\mathrm{E}\left\{\left|v_{k}^{n}\right|^{2}\right\}, \forall n \in \mathcal{N}, k \in \mathcal{K}$. At the $\nu$ th iteration, user $k$ solves the following optimization problem [2]:

$$
\begin{array}{cl}
\max _{\mathbf{s}_{\mathbf{k}}^{(\nu)}} & R_{k}^{(\nu)} \\
\text { subject to } & R_{k}^{(\nu)} \leq \sum_{n=1}^{N} \log \left(1+\frac{s_{k}^{n,(\nu)}}{I_{k}^{n,(\nu)}}\right) \quad \text { and } \\
& \mathbf{s}_{k}^{(\nu)} \in \mathcal{P}_{k}
\end{array}
$$

where $\mathbf{s}_{k}^{(\nu)} \triangleq\left[s_{k}^{1,(\nu)}, \ldots, s_{k}^{N,(\nu)}\right]$ denotes the power allocation strategy of user $k$ at the $\nu$ th iteration, $R_{k}^{(\nu)}$ denotes the sum rate that user $k$ can achieve on all subchannels when the noise and interference are Gaussian and independent, and $\mathcal{P}_{k}$ denotes its set of all feasible power allocations; i.e.

$$
\mathcal{P}_{k}=\left\{\mathbf{s}_{k} \mid \sum_{n=1}^{N} s_{k}^{n} \leq P_{k}, s_{k}^{n} \leq S_{\max , k}^{n}, \forall n \in \mathcal{N}\right\} .
$$

The solution of this problem is given by standard water-filling [9] and convergence analyses when the NI levels are assumed to be known exactly at the transmitters can be found in [1], [2], and [5].

\section{B. Quantized Iterative Water-Filling (QIWF)}

In this section, the receivers are assumed to use a finite number of bits to represent and feed the NI levels back to the transmitters. This assumption is more practical than the assumption that exact NI levels are available at the transmitters; feeding back exact NI levels is exhaustive of the resources available for communication. Using quantized NI levels instead of the exact ones, the transmitters determine their power allocations using standard IWF filling and adjust their data rates accordingly.
Suppose that the set $\mathcal{L}=\left\{L_{1}, \ldots, L_{Q}\right\}$ contains the $Q$ quantization levels from which the receiver selects the most appropriate level to quantize the observed NI levels. Upon observing $I_{k}^{n,(\nu)}$, the receiver of the $k$ th user chooses the level

$$
L_{k}^{n,(\nu)}=\arg \min _{X \in \mathcal{L}}\left|I_{k}^{n,(\nu)}-X\right|
$$

and feeds it back to its respective transmitter. This level can be expressed as

$$
L_{k}^{n,(\nu)}=I_{k}^{n,(\nu)}+e_{k}^{n,(\nu)}
$$

where $e_{k}^{n,(\nu)}$ is a quantization error term that satisfies $\left|e_{k}^{n,(\nu)}\right| \leq$ $E_{\max , k}$, where $E_{\max , k}$ is the maximum quantization error of user $k$. The values of $\left\{E_{\max , k}\right\}$ are design parameters that depend on the dynamic range of the NI levels and the number of bits used to represent them.

\section{Summary of the QIWF Algorithm}

In both the standard and the quantized IWF algorithms, the transmitters are assumed to use Gaussian signalling and to update their power allocations sequentially. In particular, transmitter $k$ is assumed to update its power allocation before transmitter $k+1$, for $k=1, \ldots, K-1$. Furthermore, it is assumed that the time interval between successive iterations spans many symbol intervals, which allows the receivers to obtain accurate estimates of the NI levels. The operation of the QIWF algorithm is as follows:

- At the $\nu$ th iteration, the receivers compute accurate estimates of their NI levels $\left\{I_{k}^{n,(\nu)}\right\}_{n=1}^{N}$ in (1) and use (4) to obtain $\left\{L_{k}^{n,(\nu)}\right\}_{n=1}^{N}$.

- The receivers feed back the quantized NI levels $\left\{L_{k}^{n,(\nu)}\right\}_{n=1}^{N}$ to their respective transmitters.

- At the $\nu$ th iteration, the $k$ th transmitter is scheduled to update its power allocation. The $k$ th transmitter replaces $\left\{I_{k}^{n,(\nu)}\right\}_{n=1}^{N}$ in (2) with $\left\{\Gamma L_{k}^{n,(\nu)}\right\}_{n=1}^{N}$, where $\Gamma \geq 1$ is a parameter that is used by the transmitters to account for the uncertainty arising from the quantization of the NI levels; see [7] and Section III-A below. The $k$ th transmitter solves (2) (with $\left\{\Gamma L_{k}^{n,(\nu)}\right\}_{n=1}^{N}$ instead of $\left.\left\{I_{k}^{n,(\nu)}\right\}_{n=1}^{N}\right)$ to determine its QIWF power allocation, $\mathbf{s}_{k}^{(\nu)}$, and its corresponding rate, $R_{k}^{(\nu)}$.

- The $k$ th transmitter uses $\mathbf{s}_{k}^{(\nu)}$ to send Gaussian signals with a rate $R_{k}^{(\nu)}$.

- At the $(\nu+1)$ th iteration, the transmitters receive $\left\{L_{k}^{n,(\nu+1)}\right\}_{n=1}^{N}$ and the $(k+1)$ th transmitter updates its power allocation.

Our goal in the next section is to establish a condition under which the quantized power allocation game has a unique Nash equilibrium to which the water-filling iterates are guaranteed to converge.

\section{The Convergence Condition of QIWF}

At the $\nu$ th iteration of the QIWF algorithm, the $k$ th user solves (2), but with the exact NI levels, $\left\{I_{k}^{n,(\nu)}\right\}$, replaced with their quantized counterparts, $\left\{L_{k}^{n,(\nu)}\right\}$. (Here, we assume that $\Gamma=1$. The case of $\Gamma>1$ is considered in Section III-A.) Similar to the standard IWF, the objective of each user in the QIWF algorithm is a continuously differentiable concave function. Hence, using Proposition 2.2.9 in [10] it can be seen that the power allocation game has at least one Nash equilibrium. Our goal now is to derive a condition under which this equilibrium is unique. When this condition is satisfied, the equilibrium power allocations and rates can be determined a priori and the communication codebooks can be designed offline. We begin by recalling the sufficient condition derived in [2] for the standard IWF algorithm to have a unique Nash equilibrium. The analysis in [2] considered the case in which the channels are static. When the channels are not static, 
i.e., time-varying, the users update their power allocations to adapt not only to other users' power allocations, but also to channel variations. In this case, the channel coefficients may change before a Nash equilibrium is reached.

Let $C_{j k}$ denote the $j k$ th entry of the matrix $C \in \mathbb{R}^{K \times K}$, where $C_{j k}=\max _{n}\left\{\alpha_{j k}^{n}\right\}, j, k=1, \ldots, K$. Let $A$ and $B$ denote the strictly lower and the strictly upper triangular parts of $C$, respectively. Using the fact that the matrix $(I-A)$ is a $Z$-matrix (i.e., its off-diagonal entries are nonpositive) [11], it was shown in [2] that if the spectral radius

$$
\rho\left((I-A)^{-1} B\right)<1
$$

the transformation that maps the error from one iteration to the next is a contraction. This contraction ensures that the error norm decays to zero at least at a geometric rate.

To derive a convergence condition for the case when quantized NI levels are fed back, we begin by making the following remark for the case when these levels are known perfectly at the transmitters.

Remark 1: As seen from (2), the transmitters do not allocate their powers based on the particular coefficients $\left\{\alpha_{i j}^{n}\right\}$, but rather on the aggregate noise and interference. However, the convergence condition in (6) and that derived herein are explicit in $\left\{\alpha_{i j}^{n}\right\}$.

We will later show how this observation will enable us to represent the quantization error using virtual channel coefficients, which will then lead us to derive a sufficient convergence condition. Further insight into the convergence behavior of the IWF algorithm can be summarized in the following remark.

Remark 2: The potential of the users not converging arises from their competitiveness and from the desire to guarantee that the rates at which they transmit their data are decodable by the respective receivers. Hence, if the receivers feed back values that do not correspond to the true NI levels, the power allocation iterations may converge irrespective of the values of $\left\{\alpha_{j k}\right\}$ (e.g., the receivers feed back a constant value). However, in that case the receivers are not guaranteed to be able to decode their received signals.

Using these remarks we will derive a sufficient convergence condition for the QIWF algorithm. At the $\nu$ th iteration of this algorithm, the transmitter of user $k \in \mathcal{K}$ water-fills on $\left\{L_{k}^{n,(\nu)}\right\}$ instead of $\left\{I_{k}^{n,(\nu)}\right\}$. To exploit the analysis in [2] in the case of QIWF, we express the levels $\left\{L_{k}^{n,(\nu)}\right\}$ as [cf. (1)]

$$
\begin{aligned}
& L_{k}^{n,(\nu)}=\sigma_{k}^{n}+\sum_{j=1}^{k-1}\left(\alpha_{j k}^{n}+q_{j k}^{n,(\nu)}\right) s_{j}^{n,(\nu)} \\
& +\sum_{j=k+1}^{K}\left(\alpha_{j k}^{n}+q_{j k}^{n,(\nu)}\right) s_{j}^{n,(\nu-1)}
\end{aligned}
$$

where $\left\{q_{j k}^{n,(\nu)}\right\}$ are virtual iteration-dependent channel coefficients. We note that, based on Remark 1, any set of $\left\{q_{j k}^{n,(\nu)}\right\}$ that satisfies (7) will exhibit the same convergence characteristics. However, for given $\left\{L_{k}^{n,(\nu)}\right\},\left\{\sigma_{k}^{n}\right\}$ and $\left\{\alpha_{j k}^{n}\right\}$, there are infinitely many choices of $\left\{q_{j k}^{n,(\nu)}\right\}$ that satisfy (7). To derive a convergence condition, we seek a deterministic map that associates with each quantization level in $\mathcal{L}$ a particular set $\left\{q_{j k}^{n}\right\}_{j=1, j \neq k}^{K}$; i.e., a map $\phi: \mathcal{L} \rightarrow \mathbb{R}^{K-1}$.

To find an appropriate map, we revisit the condition in (6). Let us construct the matrices $\hat{A}^{(\nu)}$ and $\hat{B}^{(\nu)}$ to be the matrices that correspond to $A$ and $B$ in (6), but with entries constructed from $\left\{\left(\alpha_{j k}^{n}+q_{j k}^{n, \nu}\right)\right\}$ instead of $\left\{\alpha_{j k}^{n}\right\}$. We would like to find a set of iteration-independent coefficients $\left\{q_{j k}^{n}\right\}$ for each quantization level in $\mathcal{L}$ such that the iterations are guaranteed to converge for any quantization error.

Since at any iteration $\nu$, the NI levels will be quantized to one of the quantization levels in $\mathcal{L}$, for the QIWF algorithm to converge it suffices to ensure that $\rho\left(\left(I-\hat{A}^{(\nu)}\right)^{-1} \hat{B}^{(\nu)}\right)<1$ for every level in $\mathcal{L}$. However, unless the map $\phi: \mathcal{L} \rightarrow \mathbb{R}^{K-1}$, which generates a set $\left\{q_{j k}^{n,(\nu)}\right\}_{j=1, j \neq k}^{K}$ for every $L_{k}^{n,(\nu)} \in \mathcal{L}$ is properly chosen, the convergence condition may be quite difficult to satisfy. To see this, observe that, if some of the power iterates in (7) are zero, the corresponding coefficients $\left\{q_{j k}^{n,(\nu)}\right\}$ can be arbitrary large resulting in unbounded spectral radius of the matrix $\left(I-\hat{A}^{(\nu)}\right)^{-1} \hat{B}^{(\nu)}$; cf. Lemma 2 below.

Our goal now is to find an appropriate map, and then use this map to bound $\left\{q_{j k}^{n,(\nu)}\right\}_{j=1, j \neq k}^{K}$ and subsequently to bound the spectral radius of $\left(I-\hat{A}^{(\nu)}\right)^{-1} \hat{B}^{(\nu)}$. As demonstrated earlier, despite potential convergence, without an appropriate map, $\rho\left(\left(I-\hat{A}^{(\nu)}\right)^{-1} \hat{B}^{(\nu)}\right)$ may be arbitrary large if at any iteration one of the users allocate zero power to one of the available subchannels. Hence, for $\rho\left(\left(I-\hat{A}^{(\nu)}\right)^{-1} \hat{B}^{(\nu)}\right)$ to provide an effective convergence criterion, it is desirable for the map $\phi$ to yield the set of equivalent channel parameters $\left\{q_{j k}^{n,(\nu)}\right\}_{j=1, j \neq k}^{K}$ for each quantization level in $\mathcal{L}$ that minimizes $\rho\left(\left(I-\hat{A}^{(\nu)}\right)^{-1} \hat{B}^{(\nu)}\right)$, at each iteration $\nu$. In other words, the optimal map can be regarded as the one that corresponds to the solution of following optimization problem for all iterations $\nu$ :

$$
\begin{array}{cl}
\min _{\left\{q_{j k}^{n,(\nu)}\right\}} \rho\left(\left(I-\hat{A}^{(\nu)}\right)^{-1} \hat{B}^{(\nu)}\right) \\
\text { subject to } \quad L_{k}^{n,(\nu)}=\sigma_{k}^{n}+\sum_{j=1}^{k-1}\left(\alpha_{j k}^{n}+q_{j k}^{n,(\nu)}\right) s_{j}^{n,(\nu)} \\
+\sum_{j=k+1}^{K}\left(\alpha_{j k}^{n}+q_{j k}^{n,(\nu)}\right) s_{j}^{n,(\nu-1)}, \quad \forall k, n .
\end{array}
$$

However, this optimization problem is not convex and its solution is iteration-dependent. To see this, consider the case of $K=2$. In this case, $\rho\left(\left(I-\hat{A}^{(\nu)}\right)^{-1} \hat{B}^{(\nu)}\right)=\left(\max _{n \in \mathcal{N}}\left\{\alpha_{12}^{n}+q_{12}^{n,(\nu)}\right\}\right)\left(\max _{n \in \mathcal{N}}\left\{\alpha_{21}^{n}+\right.\right.$ $\left.\left.q_{21}^{n,(\nu)}\right\}\right)$, which is not jointly convex in $\left\{q_{12}^{n,(\nu)}\right\}$ and $\left\{q_{21}^{n,(\nu)}\right\}$. Hence, even finding the optimal solution of this problem numerically is difficult, let alone finding a closed-form expression for this solution in terms of $\left\{L_{k}^{n,(\nu)}\right\}$, which is needed to proceed with the analysis.

To circumvent this difficulty, we seek insight into this problem by bounding the objective in (8a). To do so, we note that, because $\left(I-\hat{A}^{(\nu)}\right)$ is lower triangular with unity diagonal entries, $\operatorname{det}\left(\left(I-\hat{A}^{(\nu)}\right)=1\right.$. Hence, the entries of the matrix $\left(I-\hat{A}^{(\nu)}\right)^{-1} \hat{B}^{(\nu)}$ can be expressed as polynomials in $\left\{\alpha_{j k}^{n}\right\}$ with iteration-dependent coefficients $\left\{q_{j k}^{n,(\nu)}\right\}$. Now, using ([11], Problem 3.0.3), the spectral radius can be bounded by

$$
\begin{aligned}
\rho\left(\left(I-\hat{A}^{(\nu)}\right)^{-1} \hat{B}^{(\nu)}\right) \leq \min \{ & \left\|\left(I-\hat{A}^{(\nu)}\right)^{-1} \hat{B}^{(\nu)}\right\|_{\infty} \\
& \left.\left\|\left(I-\hat{A}^{(\nu)}\right)^{-1} \hat{B}^{(\nu)}\right\|_{1}\right\} .
\end{aligned}
$$

From this bound, it is seen that $\rho\left(\left(I-\hat{A}^{(\nu)}\right)^{-1} \hat{B}^{(\nu)}\right)$ is bounded by a polynomial in $\left\{\alpha_{j k}^{n}\right\}$ with coefficients $\left\{q_{j k}^{n,(\nu)}\right\}$. Since $\alpha_{j k}^{n} \geq 0$ for all $j, k$ and $n$, the polynomials on the right hand side of (9) are bounded by polynomials in which the coefficients $\left\{q_{j k}^{n,(\nu)}\right\}$ that satisfy (7) are nonnegative. In this case, the bounding polynomial is monotonically increasing in each coefficient $q_{j k}^{n,(\nu)}$. Using this observation we can find an appropriate, yet not necessarily optimal, map by solving the following problem:

$$
\begin{aligned}
\min _{\left\{q_{j k}^{n,(\nu)}\right\}} & \max _{n, j, k, j \neq q} q_{j k}^{n,(\nu)} \\
\text { subject to } & q_{j k}^{n,(\nu)} \geq 0, \quad \forall j \in \mathcal{K}-\{k\} \\
L_{k}^{n,(\nu)} & =\sigma_{k}^{n}+\sum_{j=1}^{k-1}\left(\alpha_{j k}^{n}+q_{j k}^{n,(\nu)}\right) s_{j}^{n,(\nu)} \\
& +\sum_{j=k+1}^{K}\left(\alpha_{j k}^{n}+q_{j k}^{n,(\nu)}\right) s_{j}^{n,(\nu-1)}, \quad \forall k, n .
\end{aligned}
$$


In Appendix A, this problem is cast as a linear program (LP) and one of its solutions is shown to be $q_{j k}^{n,(\nu)}=c_{k}^{n,(\nu)}, j \neq k$, where $\left\{c_{k}^{n,(\nu)}\right\}$ is a set of scalars that do not depend on $j$. Substituting for $q_{j k}^{n,(\nu)}$ in (10c) yields

$$
\begin{aligned}
L_{k}^{n,(\nu)}= & \sigma_{k}^{n}+\sum_{j=1}^{k-1} \alpha_{j k}^{n} s_{j}^{n,(\nu)}+\sum_{j=k+1}^{K} \alpha_{j k}^{n} s_{j}^{n,(\nu-1)} \\
& +c_{k}^{n,(\nu)} \sum_{j=1, j \neq k}^{K} s_{j}^{n,(\nu)} \\
= & I_{k}^{n,(\nu)}+c_{k}^{n,(\nu)} \sum_{j=1, j \neq k}^{K} s_{j}^{n,(\nu)} .
\end{aligned}
$$

Using (11), we can bound the scalars $\left\{c_{k}^{n,(\nu)}\right\}$. In particular, we have

$$
c_{k}^{n,(\nu)}=\frac{L_{k}^{n,(\nu)}-I_{k}^{n,(\nu)}}{\sum_{j=1, j \neq k}^{K} s_{j}^{n,(\nu)}} \leq \frac{E_{\max , k}}{\sum_{j=1, j \neq k}^{K} s_{j}^{n,(\nu)}} .
$$

To find an iteration-independent bound on $c_{k}^{n,(\nu)}$, we use the following result from [12].

Lemma 1: The minimum power allocated by any user $k \in \mathcal{K}$ to any subchannel $n \in \mathcal{N}$ at any iteration $\nu$ is lower bounded by $\eta_{k}^{n}$; i.e., $s_{k}^{n,(\nu)} \geq \eta_{k}^{n}$, for all $n, k, \nu$, where

$$
\begin{aligned}
\eta_{k}^{n}=\left[N ^ { - 1 } \left(P_{k}\right.\right. & \left.+\sum_{i=1}^{m_{k}} \sigma_{k}^{\pi_{k}(i)}\right) \\
& \left.\quad\left(1-N^{-1}\right) \sum_{j=0, j \neq k}^{K} \alpha_{j k}^{n} S_{\max , j}^{n}-\sigma_{k}^{n}\right]^{+},
\end{aligned}
$$

where $m_{k}$ is a lower bound on the number of subchannels occupied by user $k$ and is given by the largest integer for which $\left(m_{k}-1\right) I_{\max , k}^{\pi_{k}(r)} \leq$ $P_{k}+\sum_{i=1}^{m_{k}-1} \sigma_{k}^{(i)}$, is satisfied for all $r \leq m_{k}$. In this expression, $I_{\max , k}^{n}=\sigma_{k}^{n}+\sum_{j=1, j \neq k}^{K} \alpha_{j k}^{n} S_{\max , j}^{n}, \pi_{k}(\cdot)$ is defined to be the permutation of subchannels of user $k$ such that $I_{\max , k}^{\pi_{k}(1)} \leq I_{\max , k}^{\pi_{k}(2)} \leq \cdots \leq$ $I_{\max , k}^{\pi_{k}(N)}$, and $\sigma_{k}^{(i)}$ denotes the equivalent variance of the noise of user $k$ that satisfies $\sigma_{k}^{(i)} \leq \sigma_{k}^{(i+1)}$, for $i=1, \ldots, N-1$.

Invoking this lemma into (12), we have

$$
c_{k}^{n,(\nu)} \leq \frac{E_{\max , k}}{\sum_{j=1, j \neq k}^{K} \eta_{j}^{n}} \triangleq \xi_{k}^{n} .
$$

Using (14), we now present the main result of the paper.

Theorem 1: Let $\left[C_{Q}\right]_{j k}=\max _{n}\left\{\alpha_{j k}^{n}+\xi_{k}^{n}\right\}$ be the $j k$ th entry of the matrix $C_{Q} \in \mathbb{R}^{K \times K}$. Let $A_{Q}$ and $B_{Q}$ be the strictly lower and upper triangular parts of $C_{Q}$, respectively. Then, if

$$
\rho\left(\left(I-A_{Q}\right)^{-1} B_{Q}\right)<1
$$

the iterates of the QIWF power allocation game converge to a unique Nash equilibrium. [13].

Proof: To prove this theorem, we use the following lemma from

Lemma 2: Let $F$ and $G$ be nonnegative matrices satisfying $F \leq G$, element-wise. Then $\rho(F) \leq \rho(G)$.

From this lemma, it is seen that the spectral norm increases monotonically with the entries of any nonnegative matrix. Now, since $\operatorname{det}(I-$ $\left.\hat{A}^{(\nu)}\right)=1$, and $\left(I-\hat{A}^{(\nu)}\right)$ is a $Z$-matrix, it follows that the entries of $\left(I-\hat{A}^{(\nu)}\right)^{-1} \hat{B}^{(\nu)}$ are polynomials in $\left\{\left(\alpha_{j k}^{n}+q_{j k}^{n,(\nu)}\right)\right\}$. However, we have shown that to represent the quantization of the NI levels, it suffices to set $q_{j k}^{n,(\nu)}=c_{k}^{n,(\nu)} \leq \xi_{k}^{n}$, for all $j \neq k$. Doing so, we can write

$$
\hat{A}^{(\nu)} \leq A_{Q} \text { and } \hat{B}^{(\nu)} \leq B_{Q} .
$$

Hence, we have $\left(I-\hat{A}^{(\nu)}\right)^{-1} \hat{B}^{(\nu)} \leq\left(I-A_{Q}\right)^{-1} B_{Q}$, which, using Lemma 2, implies that if the condition in (15) is satisfied, then $\rho((I-$ $\left.\left.\hat{A}^{(\nu)}\right)^{-1} \hat{B}^{(\nu)}\right)<1$. Hence, the condition in (15) guarantees the contraction of the norm of the error vectors, and completes the proof.

Noting that $\eta_{k}^{n}$ in (13) is monotonically increasing in $P_{k}$, it can be seen from Theorem 1 that as the quantization resolution or the users' powers increase, the sufficient condition for the QIWF algorithm to have a unique Nash equilibrium approaches the corresponding condition for the standard IWF algorithm.

\section{A. Convergence of QIWF With $\Gamma>1$}

In the QIWF algorithm the transmitters allocate their powers and subsequently their rates based on quantized, rather than accurate, NI levels. Because of the inherent uncertainty associated with quantization, the rates allocated by the transmitters to some subchannels may exceed the maximum decodable rates of the receivers; cf. Remark 2. To avoid such occurrences, the transmitters may use the robust iterative water-filling strategy proposed in [7]. In this strategy, the transmitters allocate their powers and rates based on scaled versions of their received NI levels. This strategy was shown in [7] to reduce the competitiveness among users and to enable them to cooperate unintentionally. In fact, it was shown in [7] that scaling the NI levels enables the network to support rates that are close to those supported by the cooperative price-based IWF algorithm proposed in [6].

To implement the robust iterative water-filling strategy the transmitters allocate their power based on $\left\{\Gamma L_{k}^{n,(\nu)}\right\}$, rather than $\left\{L_{k}^{n,(\nu)}\right\}$, where $\Gamma$ is an appropriately chosen parameter greater than one. In this case, using an approach similar to the one used to derive Theorem 1, the following result can be proved.

Corollary 1: The QIWF with $\Gamma>1$ converges to the unique Nash equilibrium of the power allocation game if

$$
\rho\left(\left(\Gamma^{-1} I-A_{Q}\right)^{-1} B_{Q}\right)<1 .
$$

We now show that for any $\Gamma>1$, the condition in (16) is more stringent than that in (15). To do so, we note that because $\Gamma>1$, we can write $\left(\Gamma^{-1} I-A_{Q}\right)<\left(I-A_{Q}\right)$. However, both $\left(\Gamma^{-1} I-A_{Q}\right)$ and $\left(I-A_{Q}\right)$ are positive stable, in addition to being $Z$-matrices. Hence, from ([11], Theorem 2.5.4), we have $\left(\Gamma^{-1} I-A_{Q}\right)^{-1} B_{Q}>(I-$ $\left.A_{Q}\right)^{-1} B_{Q}$, which, from Lemma 2 , yields $\rho\left(\left(\Gamma^{-1} I-A_{Q}\right)^{-1} B_{Q}\right) \geq$ $\rho\left(\left(I-A_{Q}\right)^{-1} B_{Q}\right)$. This implies that the condition in (16) is generally more stringent than that in (15).

The above discussion indicates that, although using power allocation strategies based on scaled versions of the quantized NI levels can be beneficial from a network utility perspective [7], aggressive quantization and scaling may jeopardize the convergence of the power allocations.

\section{NUMERICAL EXAMPLES}

In this section, we provide two numerical examples. In the first example, we investigate the rates achieved by the QIWF algorithm and in the second example we investigate the convergence behaviour of this algorithm. In both examples, the receivers are assumed to round the NI levels to the closest integer, i.e., $E_{\max , k}=0.5$, the transmitters are assumed to have identical power budgets, $P_{k}=P$, and the spectral mask of each transmitter $S_{\max , k}^{n}=0.2 P, \forall k \in \mathcal{K}, n \in \mathcal{N}$. The noise variance $N_{0}=1$.

Example 1: In this example, we consider a case with $K=4$ users and $N=16$ subchannels. The subchannels are assumed to be frequency flat Rayleigh fading with coefficients, $\left\{h_{j k}^{n}\right\}$, drawn from the 


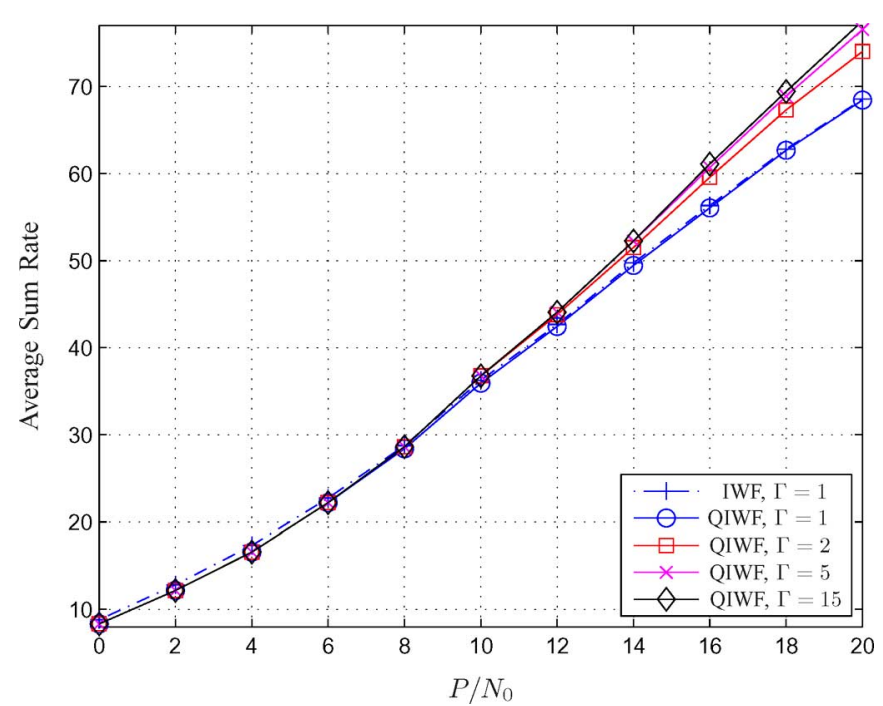

Fig. 1. Average sum rate achieved by standard and quantized IWF for $\Gamma=1$, 2,5 , and 15 .

TABLE I

ChANNEl COEFFICIENTS USED IN EXAMPLE 2

\begin{tabular}{c|ccc|ccc|} 
& \multicolumn{3}{|c|}{$\left|h_{j k}^{1}\right|^{2}$} & \multicolumn{3}{|c|}{$\left|h_{j k}^{2}\right|^{2}$} \\
\hline & user 1 & user 2 & user 3 & user 1 & user 2 & user 3 \\
\hline user 1 & 12.799 & 0.027 & 0.042 & 0.810 & 0.060 & 1.526 \\
user 2 & 0.378 & 2.272 & 0.281 & 0.691 & 4.264 & 0.035 \\
user 3 & 0.756 & 0.118 & 16.304 & 0.509 & 1.256 & 4.394 \\
\hline & \multicolumn{3}{|c|}{$\left|h_{j k}^{3}\right|^{2}$} & & & $\left|h_{j k}^{4}\right|^{2}$ \\
\hline user 1 & 0.736 & 0.084 & 0.108 & 1.935 & 0.018 & 0.201 \\
user 2 & 0.652 & 3.090 & 0.047 & 0.176 & 2.510 & 0.752 \\
user 3 & 0.297 & 0.431 & 6.289 & 0.262 & 0.048 & 15.746 \\
\hline & \multicolumn{3}{|c|}{$\left|h_{j k}^{5}\right|^{2}$} & & & $\left|h_{j k}^{6}\right|^{2}$ \\
\hline user 1 & 5.302 & 0.262 & 0.180 & 4.123 & 0.247 & 0.668 \\
user 2 & 0.209 & 3.805 & 0.160 & 0.765 & 8.649 & 0.021 \\
user 3 & 1.552 & 0.692 & 1.186 & 0.765 & 0.453 & 2.395 \\
\hline
\end{tabular}

standard zero mean and unit variance circularly symmetric complex Gaussian distribution.

In Fig. 1 the average sum rate achieved by the standard IWF algorithm and the QIWF algorithm with $\Gamma=1,2,5$ and 15 are plotted versus $P / N_{0}$. From this figure, it can be seen that when $\Gamma=1$, the average sum rate achieved by QIWF is close to that achieved by the standard IWF algorithm. However, by increasing $\Gamma$, a slight deterioration is observed at low $P / N_{0}$, but a significant gain is observed at higher $P / N_{0}$. For instance, at $P / N_{0}=20 \mathrm{~dB}$, the average sum rate achieved by QIWF with $\Gamma=5$ is about 77 bits per channel use (bpcu), whereas that achieved by the standard IWF is about 68 bpcu. Hence, it can be seen that QIWF with $\Gamma>1$ can yield significant rate gains. This result is consistent with the analysis in [7], wherein it was shown that increasing $\Gamma$ reduces the competitiveness among users.

Example 2: In this example we investigate the convergence behaviour of the QIWF algorithm. We consider a scenario with $K=3$ users and $N=6$ subchannels with the randomly selected coefficients in Table I. In this instance, the channel coefficients $\left\{h_{j k}^{n}\right\}_{j \neq k}$ are drawn from the standard zero mean unit variance complex Gaussian distribution, whereas the channel coefficients $\left\{h_{k k}^{n}\right\}_{k=1}^{K}$ are drawn from the zero mean complex Gaussian distribution with variance 10 .

From this table, it can be verified that $\rho\left((I-A)^{-1} B\right)=0.43<$ 1 , which implies that the standard IWF algorithm has a unique Nash equilibrium; cf. (6). To verify this result, in Figs. 2(a) and (b) the norm of the error $\sum_{k=1}^{K}\left\|\mathbf{s}_{k}^{(\nu+1)}-\mathbf{s}_{k}^{(\nu)}\right\|$ is plotted versus the iteration index, $\nu$, when $P=10 \mathrm{~dB}$ and $P=20 \mathrm{~dB}$, respectively.

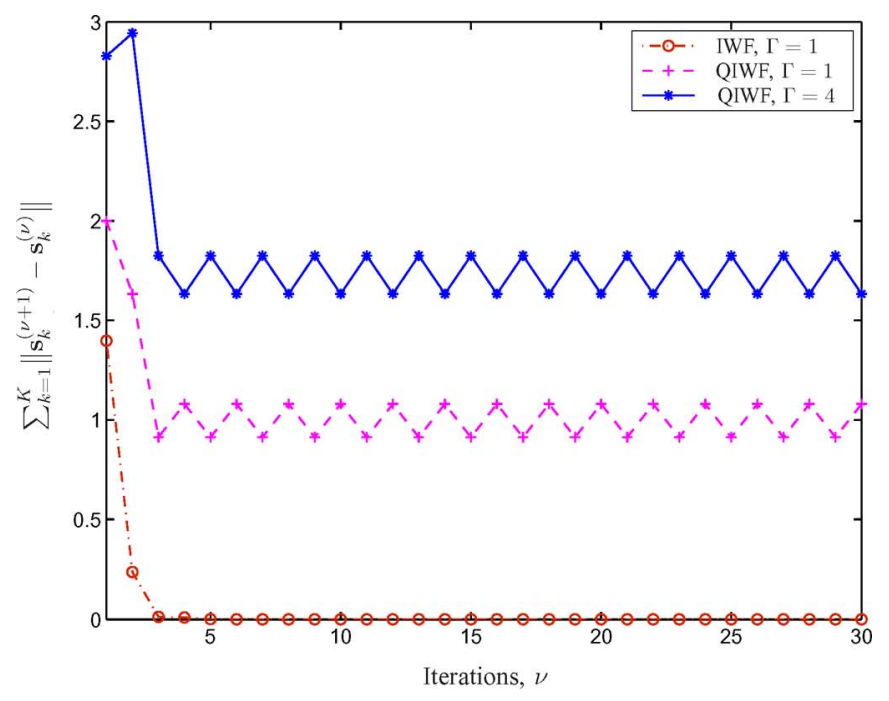

(a)

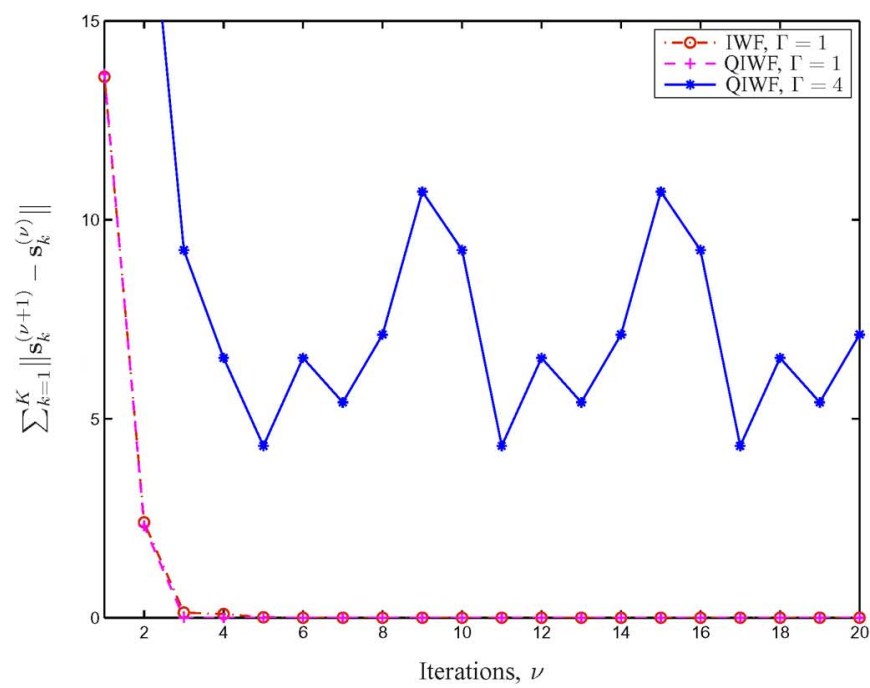

(b)

Fig. 2. Convergence behaviour of QIWF with $\Gamma=1,4$. (a) $P=10 \mathrm{~dB}$. (b) $P=20 \mathrm{~dB}$.

When no quantization is used, it can be seen from Figs. 2(a) and (b), that, because the condition in (6) is satisfied, the standard IWF with $\Gamma=1$ converges, irrespective of the power level. Now, we consider the case in which the NI levels are quantized. When $P=10 \mathrm{~dB}$, the QIWF algorithm with $\Gamma=1$ does not converge. However, from the discussion following Theorem 1, we noted that the QIWF algorithm is guaranteed to converge if (6) is satisfied, and $P$ is sufficiently high. This observation is verified in Fig. 2(b), which shows that by increasing $P$ to $20 \mathrm{~dB}$, the iterates of the quantized IWF with $\Gamma=1$ converge. In contrast, for the case of QIWF with $\Gamma=4$, the condition in Corollary 1 is not satisfied and the iterates do not converge at both $P=10 \mathrm{~dB}$ and $P=20 \mathrm{~dB}$.

\section{CONCLUSION}

In this paper we investigated the convergence of the IWF algorithm when the noise-plus-interference (NI) levels fed back from the receivers are quantized. In this case, we have shown that at least one Nash equilibrium exists for the power allocation game. We then derived a sufficient condition under which this equilibrium is unique, and the iterates are guaranteed to converge. Using this condition we showed 
that quantizing the NI levels can cause the, otherwise convergent IWF algorithm, to oscillate. Furthermore, we showed that if the standard IWF algorithm converges and the transmit power is sufficiently high, the QIWF algorithm is guaranteed to converge. Finally, we showed that although scaling the NI levels can increase the network utility, it can jeopardize the convergence of the QIWF algorithm.

\section{APPENDIX A}

\section{AN OPtimal SOLUTION OF (10)}

The optimization in (10) can be cast as

$\min t$

$$
\begin{gathered}
\text { subject to } \quad q_{j k}^{n,(\nu)} \leq t, \quad j, k \in \mathcal{K}, j \neq k, n \in \mathcal{N} \\
\sum_{j=1, j \neq k}^{K} a_{j}^{n,(\nu)} q_{j k}^{n,(\nu)}=b_{k}^{n,(\nu)} \\
k=1, \ldots K, \quad n=1, \ldots, N
\end{gathered}
$$

where for notational convenience, we have used

$$
\begin{aligned}
& a_{j}^{n,(\nu)}=\left\{\begin{array}{ll}
s_{j}^{n,(\nu)} & j \in\{1, \ldots, k-1\} \\
s_{j}^{n,(\nu-1)} & j \in\{k+1, \ldots, K\}
\end{array} \quad\right. \text { and } \\
& b_{k}^{n,(\nu)}=L_{k}^{n,(\nu)}-\sigma_{k}^{n}-\sum_{j=1, j \neq k}^{K} \alpha_{j k}^{n} a_{j}^{n,(\nu)}, \quad \forall k, n, \nu .
\end{aligned}
$$

The optimization in (17) is in the form of an LP, for which the Karush-Kuhn-Tucker (KKT) conditions are necessary and sufficient for optimality. Using $\left\{\lambda_{j k}^{n,(\nu)}\right\}$ and $\left\{\mu_{k}^{n,(\nu)}\right\}$ to denote the Lagrange multipliers corresponding to the constraints in (17b) and (17c), respectively, the Lagrangian can be expressed as

$$
\begin{aligned}
L=t+\sum_{n=1}^{N} \sum_{k=1}^{K} & \sum_{j=1, j \neq k}^{K} \lambda_{j k}^{n,(\nu)}\left(q_{j k}^{n,(\nu)}-t\right) \\
& +\sum_{n=1}^{N} \sum_{k=1}^{K} \mu_{k}^{n,(\nu)}\left(\sum_{j=1, j \neq k}^{K} a_{j}^{n,(\nu)} q_{j k}^{n,(\nu)}-b_{k}^{n,(\nu)}\right) .
\end{aligned}
$$

From the KKT conditions, we have $\frac{\partial L}{\partial t}=1-$ $\sum_{n=1}^{N} \sum_{k=1}^{K} \sum_{j=1, j \neq k}^{K} \lambda_{j k}^{n,(\nu)}=0$, which yields

$$
\sum_{n=1}^{N} \sum_{k=1}^{K} \sum_{j=1, j \neq k}^{K} \lambda_{j k}^{n,(\nu)}=1
$$

This equation implies that at least one element of the set $\left\{\lambda_{j k}^{n,(\nu)}\right\}$ is strictly positive. From the KKT conditions, we also have that

$$
\frac{\partial L}{\partial q_{j k}^{n,(\nu)}}=\lambda_{j k}^{n,(\nu)}+\mu_{k}^{n,(\nu)} a_{j}^{n,(\nu)}=0 .
$$

The last equality implies that for any $k, n$ and $\nu$ for which $a_{j}^{n,(\nu)} \mu_{k}^{n,(\nu)} \neq 0$ for $j=1, \ldots, K, j \neq k$

$$
\lambda_{j k}^{n,(\nu)}>0, \quad j=1, \ldots, K, \quad j \neq k .
$$

Notice that from the KKT conditions, the multipliers $\lambda_{j k}^{n,(\nu)}$ must be nonnegative. From the KKT complementarity slackness conditions we have

$$
\lambda_{j k}^{n,(\nu)}\left(q_{j k}^{n,(\nu)}-t\right)=0, \quad \forall j, k, n, \nu .
$$

Hence, from (19) and (20), we have that, when $a_{j}^{n,(\nu)} \mu_{k}^{n,(\nu)} \neq 0$

$$
q_{j k}^{n,(\nu)}=c_{k}^{n,(\nu)}=\frac{b_{k}^{n,(\nu)}}{\sum_{j=1, j \neq k}^{K} a_{j}^{n,(\nu)}}, \quad j=1, \ldots, K, j \neq k .
$$

When $a_{j}^{n,(\nu)} \mu_{k}^{n,(\nu)}=0$, the equality in (18) yields $\lambda_{j k}^{n,(\nu)}=0$, which implies that the corresponding inequalities in (17b) are not necessarily active. However, the KKT conditions are still satisfied by the choice of $q_{j k}^{n,(\nu)}$ in (21).

\section{ACKNOWLEDGMENT}

The authors would like to thank C. Bontu, C. Royer, and J. Womack of Research In Motion for their valuable comments on the manuscript. The authors would also like to thank the anonymous reviewers for their careful reading and constructive remarks.

\section{REFERENCES}

[1] W. Yu, G. Ginis, and J. M. Cioff, "Distributed multiuser power control for digital subscriber lines," IEEE J. Sel. Areas Commun., vol. 20, no. 5, pp. 1105-1115, Jun. 2002.

[2] Z.-Q. Luo and J.-S. Pang, "Analysis of iterative waterfilling algorithm for multiuser power control in digital subscriber lines," EURASIP Appl. Signal Process., vol. 2006, pp. 1-10, Apr. 2006.

[3] G. Scutari, D. P. Palomar, and S. Barbarossa, "Optimal linear precoding strategies for wideband noncooperative systems based on Game Theory—Part I: Nash equilibria," IEEE Trans. Signal Process., vol. 56, no. 3, pp. 1230-1249, Mar. 2008.

[4] S. Boyd and L. Vandenberghe, Convex Optimization. Cambridge, U.K.: Cambridge Univ. Press, 2004.

[5] G. Scutari, D. P. Palomar, and S. Barbarossa, "Asynchronous iterative waterfilling for Gaussian frequency-selective interference channels," IEEE Trans. Inf. Theory, vol. 54, no. 7, pp. 2868-2878, Jul. 2008.

[6] F. Wang, M. Krunz, and S. Cui, "Price-based spectrum management in cognitive radio networks," IEEE J. Sel. Topics Signal Process., vol. 2 no. 1 , pp. $74-87$, Feb. 2008

[7] R. H. Gohary and T. J. Willink, "Robust IWFA for open-spectrum communications," IEEE Trans. Signal Process., vol. 57, no. 12, pp. 4964-4970, Dec. 2009.

[8] M. Hong and A. Garcia, "Averaged iterative water-filling algorithm: Robustness and convergence," IEEE Trans. Signal Process., vol. 59, no. 11 , pp. 2448-2454, Nov. 2011

[9] T. M. Cover and J. A. Thomas, Elements of Information Theory. New York: Wiley, 1991.

[10] F. Facchinei and J. Pang, Finite-Dimensional Variational Inequalities and Complementarity Problem. New York: Springer-Verlag, 2003

[11] R. A. Horn and C. R. Johnson, Topics in Matrix Analysis. Cambridge, U.K.: Cambridge Univ. Press, 1994.

[12] R. H. Gohary, Y. Huang, Z.-Q. Luo, and J.-S. Pang, "A generalized iterative water-filling algorithm for distributed power control in the presence of a jammer," IEEE Trans. Signal Process., vol. 57, no. 7, pp. 2660-2674, Jul. 2009.

[13] Y. A. Al'pin and L. Y. Kolotilina, "The generalized monotonicity property of the Perron root," J. Math. Sci., vol. 141, no. 6, pp. 1576-1585, 2007. 